Review Article

\title{
SURGICAL TREATMENT OF ADVANCED GASTRIC CANCER
}

\author{
Nebojša Ignjatovici ${ }^{1,2}$, Goran Stanojević $^{1,2}$, Miroslav Stojanović $^{1,2}$, Ljiljana Jeremić $^{1,2}$, Milica Nestorović $^{1,2}$, \\ Vesna Brzački $^{1,2}$, Daniela Benedeto Stojanov ${ }^{1,2}$, Miodrag Djordjević ${ }^{2,3}$, Mirjana Marinković ${ }^{2}$ \\ ${ }^{1}$ Clinic of Digestive Surgery, Clinical Center Niš, Niš, Serbia \\ ${ }^{2}$ University of Niš, Faculty of Medicine, Niš, Serbia \\ ${ }^{3}$ Clinic for Endocrine and Breast Surgery, Clinical Center Niš, Niš, Serbia
}

\begin{abstract}
Patients with advanced gastric cancer generally have poor overall prognosis as well as survival rate. Unfortunately, in the West, gastric cancer typically occurs at an advanced stage and many of these patients have tumor invasion into adjacent structures (International Union Against Cancer [UICC]/American Joint Committee on Cancer [AJCC] Stage T4). Although T4 gastric cancer patients often have peritoneal dissemination or distant metastasis, many do not have MI disease and are therefore candidates for surgery with the curative intent. A multivisceral resection (MVR) or gastrectomy with resection of adjacent organs is needed in T4 gastric cancer patients to achieve an RO resection that is one of the most powerful forecasters of gastric cancer surgery results. Spleen, distal pancreas, liver, and large intestine (mostly transverse colon) were the most commonly resected organs. The therapeutic choice with acceptable postoperative morbidity and mortality rates in locally advanced patients with gastric cancer should be gastrectomy with MVR, where complete resection could be realistically obtained and where metastatic involvement of the lymph node is not evident. MVR is done with a curative $R 0$ resection to provide advanced gastric cancer patients with the best survival chance. It was found that resections involving the pancreas, transverse colon and liver were associated with increased survival rate in comparison to MVR with resection of other structures. It was shown that survival rate significantly decreased in patients who had undergone MVR without complete resection compared to those who had an RO resection. Nevertheless, the extent of the surgical resection required and further advantages of MVR are disputable.
\end{abstract}

Key words: advanced gastric cancer, gastrectomy, multivisceral resection.

\section{Introduction}

Gastric cancer remains the world's second-largest cause of cancer-related death [1,2], including $10 \%$ of newly diagnosed cancers [2]. While gastric cancer pathogenesis and etiology are a prevailing research topic with no proven definitive mechanism to this day, many well described risk factors exist. Intestinal metaplasia, gastric adenomatous polyps, helicobacter pylori, pernicious anemia, giant hypertrophic gastritis (Ménétrier disease) and chronic atrophic gastritis are known to be associated with gastric cancer [3]. Unlike Western patients, Asian gastric cancer patients have a better prognosis due to earlier detection of the disease through frequent screening programs. The overall disease survival rate in Japan is over $70 \%$. On the other hand, over two thirds of gastric cancers in the United States as well as in Europe are usually discovered with a locally advanced resectable disease in advanced stages, since routine gastric cancer screening is not recommended due to its cost. Consequently, a 5-year survival rate of merely $25 \%$ for locally advanced disease is recorded in these countries [4].

Correspondence to: Nebojša Ignjatović, M.D., Ph.D. Faculty of Medicine, 81 Zoran Đinđić Blvd., 18000 Niš, Serbia Phone: +381638220622 E-mail: n.ignjat@gmail.com Received May $7^{\text {th }}, 2019$

\section{Histopathology Classification}

Two major types of gastric cancer, intestinal and diffuse, were characterized by Laurèn in 1965. Metastasis patterns differ from the two types of gastric cancer. The diffuse tumors show a wider spread than intestine tumors. Furthermore, in diffuse cases, lymphatic permeation of the lungs, tumors of Krukenberg and peritoneal metastases are more common. However, the liver is more commonly involved in the intestinal cancer type. Gastric adenocarcinoma rarely reports intestinal metastases. Primary stomach tumors with intestinal metastases are generally poorly differentiated with or without ring-cell differentiation of the signet and most often belong to the scirrhous type. These metastases rarely include the liver and are often associated with peritoneal seeding. There were three reported cases of multiple colonic metastases of poorly differentiated gastric adenocarcinoma presenting as colonic polyposis. In one case, multiple flat elevated lesions appeared in a rare form of gastric signet ring-cell cancer with metastases to the colon [5].

In research in determining tumor stage in gastric cancer, representing the most important independent prognostic factor, as well as for clinical practice, the American Joint Committee on Cancer (AJCC)/Union for International Cancer Control (UICC) tumor, node, metastasis (TNM) staging system has been used [4]. 
Determining the prognosis is highly influenced by the final pathological stage that follows the curative surgery. In patients with pathological stages II, III and $\mathrm{IV}$, survival rate is significantly lower [6].

\section{Optimal Surgery Treatment}

\section{The type of gastrectomy}

The most important curability factor is surgical resection [7]. The site of the primary tumor with the resection margin of a minimum of $5 \mathrm{~cm}$ from the edge of the tumor determines the type of gastrectomy. According to the Lauren classification, total gastrectomy is used to treat the 'diffuse' type tumors which are likely to spread laterally. It might not be necessary for distal types of tumors in case that mapping biopsies, careful radiological review, adequate staging, on-table Oesophago-Gastro-Duodenoscopy (OGD) with or without frozen section provide satisfactory results. Tumors of the gastric antrum (distal third cancers) need a subtotal $(80 \%)$ gastrectomy, with the excision of regional lymphatic tissue and the division of the left gastric vein and artery. Total gastrectomy is performed when sub mucosal tumor infiltration is within $7-8 \mathrm{~cm}$ of GOJ or in case of a large distal third tumor. In cases of palliation or in the very elderly, only limited gastric resections are recommended. There is no oncological advantage but increased morbidity in resecting spleen and distal pancreas for a cancer in the distal two-third of stomach. Tumors of the gastric body (middle third cancers) usually need a total gastrectomy, since it depends on the proximal tumor margin. The stomach level that remains below GOJ should be at least $2 \mathrm{~cm}$. A $7 \mathrm{~cm}$ margin from GOJ is required in serosa negative cancer cases, while $8 \mathrm{~cm}$ margin from GOJ is standard in serosa positive cancer. The surgery's overall goal is appropriate lymphadenectomy (formal D2 and posterior mediastinal, periesophageal nodes), adequate local clearance, as well as low morbidity uncomplicated anastomosis. In patients with tumors of proximal stomach located on greater curvature/ posterior wall of stomach close to splenic hilum where incidence of splenic hilar nodal involvement is likely to be high, splenic and hilar node resection are considered [6].

\section{Extension of gastric resection}

T1 disease is present at $>80 \%$ of gastric cancer patients and merely $40 \%$ of early gastric cancer are related to symptoms. Overall, $85 \%$ patients have lymph node metastases, $65 \%$ patients present as advanced cancers (T3, T4), and $40 \%$ are metastatic. Since this is a locoregional disease, the primary surgical aim is to remove the primary tumor with clear longitudinal and circumferential resection margin, with resection of associated lymph nodes and combined organ resection as required (R0 resection), following the restoration of intestinal and biliary continuity to allow adequate nutritional intake [6].

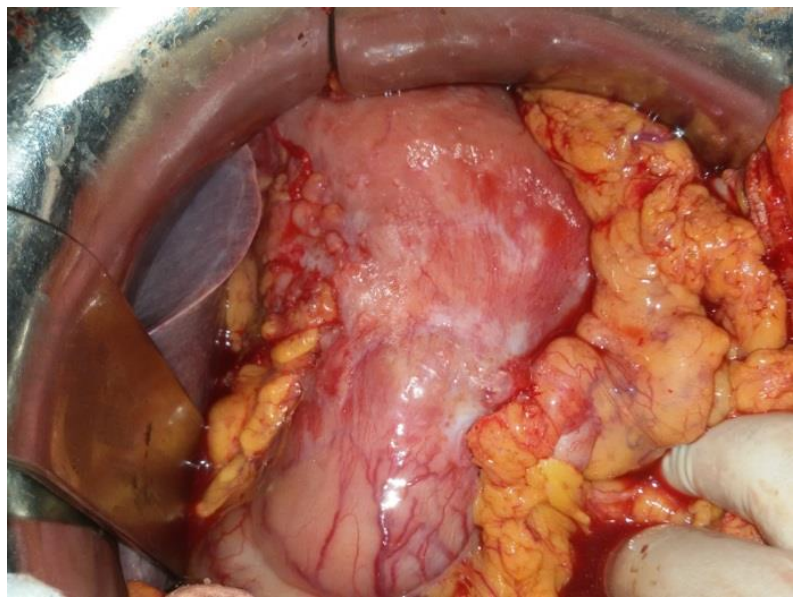

Fig. 1 Intraoperative view of advanced gastric cancer

Patients with advanced gastric cancer (Fig. 1) benefit from curative resection and an R0 resection is mainly related to improved survival rate. Some researches claim that multivisceral resection (MVR) can be done in adequately selected patients with acceptable morbidity and mortality. The most frequently resected organs were liver, distal pancreas, spleen, and large bowel (mostly transverse colon). Other frequently resected organs included gallbladder and small bowel. The organs that were less frequently resected were adrenal gland, diaphragm, pericardium, mesocolon, kidney, lung, ovary and uterus. In gastrectomy patients with MVR, the perioperative mortality rates were from 1.9 to $15.0 \%$, with five-year survival rates of $0-40 \%$. These researches were done with the aim to identify predictors of longterm survival, including the number of resected organs, UICC/AJCC stage, margin positivity and other factors, which may play a role in adequate patient selection for MVR. In order to provide patients with advanced gastric cancer the best chance at survival with a curative R0 resection, MVR is performed. Survival rate in MVR without a complete resection patients was shown to be significantly lower in comparison to those who underwent an R0 resection. Poor outcomes appear to be linked to both microscopic and macroscopic positive margins [8].

Despite the fact that a retrieval of a minimum of 16 lymph nodes and a surgical R0 resection are mandatory surgical principles, there is still some polemics on lymphadenectomy and the extent of surgical resection. The primary tumor removal is based on the location, extension and histologic subtype of gastric cancer [4]. R0 resection (negative microscopic and macroscopic margins) is a powerful forecaster of outcome for curative gastric cancer surgery patients. Unfortunately, gastric cancer is generally found at an advanced stage, and tumor invasion into adjacent structures (International Union Against Cancer [UICC]/American Joint Committee on Cancer [AJCC] Stage T4) is present in most of the patients in the West.

Even though T4 gastric cancer patients will often show peritoneal dissemination or distant metastases, 
many of them do not have M1 disease, which makes them suitable candidates for the surgery with the curative intent. A MVR, or gastrectomy with resection of adjacent organs is needed to achieve an R0 resection in patients with $\mathrm{T} 4$ gastric cancer [8]. On the other hand, many patients suffer from the incurable, advanced stage gastric cancer. The primary objective of palliative resection for this type of patients is relief of symptoms such as tumor bleeding, obstruction, or perforation. Studies have shown that surgical resection for stage IV gastric cancer can be done with low operative mortality and acceptable morbidity rates. It also provides good symptomatic relief for the patient [7].

Treating patients who are at stage IV disease includes the following: systemic chemotherapy, surgical bypass procedures, palliative resection, endoscopic interventions, investigational therapies, or best supportive care. Only modest survival rates have been recorded in patients treated with best supportive care alone. Palliative resection for stage IV gastric cancer is nowadays not a standard part of care, even though it was practiced regularly in the past. Certain previous researches have shown a benefit to the stage IV gastric cancer patient in case of palliative resection. On the other hand, palliative resection also brings the risk of surgical morbidity and prolonged hospitalization. This might reduce instead of improve the quality of life and survival rate of this type of cancer patients [9].

At the moment, the general opinion is that "a proximal margin of at least $3 \mathrm{~cm}$ is recommended for T2 or deeper tumors with an expansive growth pattern and 5 $\mathrm{cm}$ is recommended for those with infiltrative growth pattern", which puts an end to a long-term dispute. However, in T4 cases, a MVR (MVR), or gastrectomy with resection of adjacent organs is sometimes needed to provide patients with advanced gastric cancer the best survival chance with a curative R0 resection, but the prognostic benefit of MVR in patients with locally advanced disease and the postoperative morbidity are still under debate [4]. Kasakura et al. [10] found a higher complication rate in MVR group in comparison to gastrectomy alone group, with no difference in survival rates. Pacelli et al. found in their Italian multicenter observational study no important differences in mortality rates and postoperative morbidity [11]. Other researches show a survival disadvantage for gastrectomy with additional organ resection. On the contrary, most researchers found an overall 5-year survival improvement (19.9\%e38\%) for gastrectomy with MVRs patients when compared with gastrectomy or palliative surgery patients. In patients with $\mathrm{T} 4$ gastric cancer undergoing curative $\mathrm{R} 0$ resection, the 5-year survival rate ranges from $23 \%$ to $46 \%$. It decreases in cases of $\mathrm{R}+$ resection, ranging from $17.5 \%$ to $0 \%$. Colectomy, splenectomy, pancreatosplenectomy, or any other organ resection did not forecast poor survival. In locally advanced gastric cancer patients where a complete resection could be realistically obtained and when lymph node metastatic involvement is not evident, the gastrectomy with MVR should be the therapeutic choice, with an acceptable postoperative morbidity and mortality rates (4). Even though radical resection has been found to be closely connected to long-term survival for gastric cancer patients, curative resection for locally advanced gastric cancer, defined as T4 in which the tumor invades adjacent structures (T4b) or perforates serosa (T4a) was related to increased postoperative morbidity and mortality. Due to early detection of gastric cancer and improved surgical technique, the gastric cancer patients' prognosis has significantly improved. On the other hand, T4 gastric carcinoma patients' prognosis remained poor. It is therefore paramount to clarify the incidence of postoperative morbidity and mortality in patients with T4 gastric cancer who opt for curative surgeries and to determine the prognostic factors in these populations [12]. Gastrectomy with D2 lymphadenectomy (resection of perigastric lymph nodes and nodes along the named branches of the celiac axis) is considered standard surgical procedure for the advanced gastric cancer and most early-stage gastric cancers [3].

\section{The extent of gastrectomy}

The extent of gastrectomy being performed (i.e. total, subtotal/distal, or proximal gastrectomy) defines the extent of lymphadenectomy. Most commonly, a D2 dissection for a total gastrectomy would involve retrieval of lymph node stations 1-12 with a concomitant distal pancreatectomy and splenectomy. Moreover, a D1 dissection would require the perigastric nodes at stations. Lately, a modified approach to a D2 dissection by sparing the spleen and pancreas unless directly involved with the primary tumor was proposed. This technique of sparing the spleen and pancreas has shown adequate retrieval of lymph nodes without the morbidity associated with multi-visceral resection (3).

D1 lymphadenectomy happens when all N1 nodes (perigastric nodes closest to primary) are removed en bloc with the stomach (limited). D2 includes systematical removal of all N1 and N2 (distant perigastric nodes and nodes along main arteries supplying stomach) en bloc with stomach. Figure 2. Gastric cancer often remained localized to stomach and adjacent lymph node. This supports the Japanese view that radical systemic D2 lymphadenectomy has increased survival benefits. The present European description of D2 lymphadenectomy includes the removal of $>15$ lymph nodes, irrespective of node stations. Moreover, extended D3 lymphadenectomy represents a radical en bloc resection with N3 nodes outside normal lymphatic pathways from stomach, included in advanced stages station (hepatoduodenal ligament) or by retrograde lymphatic flow due to blockage of normal pathways [8]. The Japanese researchers have supported the claim that D2 resection should be used in all patients with invasive gastric carcinoma, including early gastric cancer patients. They also argued that a D3 lymphadenectomy should be performed in all patients with advanced gastric cancer with 

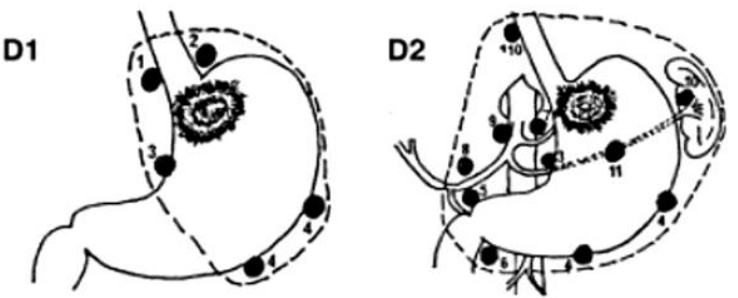

gastric cancer of the upper third of the stomach
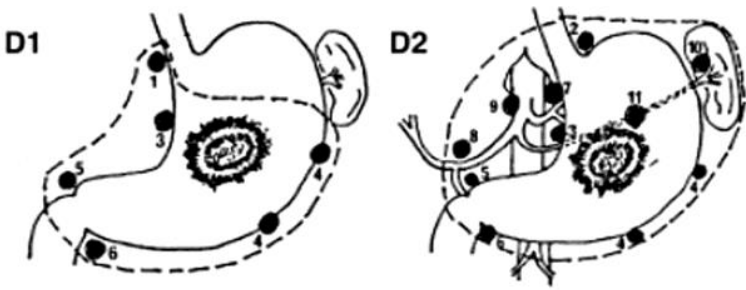

gastric cancer of the middle third of the stomach
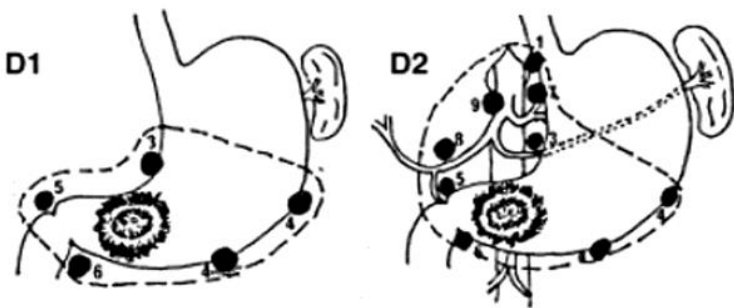

gastric cancer of the lower third of the stomach

Fig. 2 Lymphadenectomy range depending on the localisation of the gastric cancer

serosal invasion [1]. Two independent factors associated with survival were total lymph node count and number of positive lymph nodes. In patients who had more than $15 \mathrm{~N} 2$ nodes and $20 \mathrm{~N} 3$ nodes examined, a significant survival benefit was recorded. The pathologic assessment of at least 15 nodes is considered standard of care. Also, D2 lymphadenectomy is recommended, even though there is no general rule on the level of dissection required (D1 vs. D2) in the U.S. Higher post-operative mortality $(13 \%$ vs. $6.5 \%, \mathrm{P}=0.04)$ was shown in one of the Western studies and morbidity rates (46\% vs. $28 \%$, $\mathrm{P}<0.01)$ in the D2 lymphadenectomy group. There was a higher chance of undergoing concomitant pancreatectomy and splenectomy. Anastomotic complications in the D2 dissection group have shown significantly higher rates, also including severe pancreatic fistula, pancreatitis, and gastric remnant necrosis. There was no difference in overall survival, gastric cancer related deaths, or recurrence-free survival in long-term results. On the other hand, recent studies from the East and West indicate improved morbidity and mortality if routine splenectomy and pancreatectomy is avoided, in comparison to traditional D2 resection [3]. Cuschieri et al. [13] evaluated 400 patients randomized to a D1 or D2 lymphadenectomy and found a significant survival difference between patients with gastrectomy and splenectomy or pancreaticosplenectomy compared to gastrectomy alone, with no regard to the extent of lymphadenectomy.

\section{Survival and Complication}

When it comes to locally advanced gastric cancer, particularly if the adjacent organ is invaded (T4), the prognosis will be poor since a high incidence of postoperative morbidity and mortality is reported in studies, and the curative resection itself is difficult [1]. For many years, the benefits of extended organ resection for advanced gastric cancer have been discussed. This debate was recently sparked by the results of both the United Kingdom Medical Research Council and the Dutch trials evaluating the survival benefit of extended lymphadenectomy. A significant survival disadvantage in patients who have undergone gastrectomy with splenectomy or pancreaticosplenectomy was reported by both of these randomized control trials. The Medical Research Council study and the Dutch trial found that a higher complication rate, higher mortality and longer hospital stay are closely related to extended organ resection. The potential advantage of extended resection for clinical T4N0 gastric adenocarcinoma is necessary to improve the $\mathrm{R} 0$ resection rate of these lesions. The observed increase in the morbidity and mortality rates, with low survival benefits fuel the arguments against this approach. In patients undergoing gastrectomy alone, the complication rates of additional organ resection with gastrectomy have been reported to be high. When it comes to patients undergoing splenectomy, both overall complications and infectious complications have been reported. The increase in overall complications and infectious complications is the suggested reason for the decrease in overall survival. This is why performing additional organ resection in patients with $\mathrm{T} 4$ disease has been scrutinized. With minimal perioperative mortality (4\%), gastrectomy with additional organ resection for gastric cancer can be realized. In this patient subset, long-term survival can be reached, with a 3-year survival rate of $47 \%$. The biology of the primary lesion (i.e., depth of invasion and nodal stage) might forecast overall survival in this patient group. Gastrectomy with additional organ resection can be done with low mortality and acceptable morbidity if careful patient selection is implemented. In order to minimize unnecessary organ resections for early-stage disease, certain improvements should be made in preoperative evaluation to confirm T3 and T4 disease. The most powerful forecasters of survival following an $\mathrm{R} 0$ resection are depth of invasion and presence and extent of lymph node metastasis. With the understanding that the majority will be T3, gastrectomy with additional organ resection should be limited to clinically T4 tumors [14].

It cannot be said with certainty that gastrectomy alone, when yielding an R1 or R2 margin, is appropriate when R0 resection with MVR is feasible and safe. Just a few studies examined survival in relation to the type of organs resected. On the other hand, resections involving the transverse colon and the liver have been shown to be related to the increased survival compared to MVR with resection of other adjacent structures. The aim of $\mathrm{R} 0$ resection must take into account whether gastric cancer 
is actually invading adjacent organs. MVR should be reserved for T4 lesions, with true histological invasion into adjacent organs, given the risk of morbidity. Adhesions secondary to desmoplastic reaction can resemble a local invasion, especially when the pancreas is involved. MVR might include resection of two or more organs in pursuit of negative margins. Four studies studied the number of organs involved or resected as a survival forecaster. Also, Martin et al. found an increase in operative complications with lower 5-year survival while comparing one-, two-, and three-organ resections in addition to gastrectomy [8].

\section{Conclusion}

The only curative surgery for non-metastatic gastric cancer is surgical resection with extended lymph node dissection. In patients with locally advanced gastric

\section{References}

1. Van de Velde CJH. Gastric cancer: staging and surgery. Ann Oncol 2002; 13 Suppl 4:1-6.

2. Kim JH, Jang YJ, Park SS, Park SH, Kim SJ, Mok YJ, Kim CS. Surgical outcomes and prognostic factors for T4 gastric cancers. Asian J Surg 2009; 32:198-204.

3. Yamamoto M, Rashid OM, Wong J. Surgical management of gastric cancer: the East vs. West perspective. J Gastrointest Oncol 2015; 6:79-88.

4. Marano L, Polom K, Patriti A, et al. Surgical management of advanced gastric cancer: An evolving issue. Eur J Surg Oncol 2016; 42:18-27.

5. Lee HC, Yang MT, Lin KY, Tu HY, Zhang TA, Chen PH. Metastases from gastric carcinoma to colon in the form of multipleflat elevated lesions: a case report. Kaohsiung J Med Sci 2004; 20:552-527.

6. Weledji EP. How surgery can improve multimodal treatment of gastric cancer. In: Management of Gastric Cancer. Group SM, www.smgebooks.com. 2016: 1-12. https://smjournals.com/ ebooks/management-of-gastric-cancer/chapters/MGC-16-04

7. Ikeguchi M, Kader A, Takaya S, et al. Treatment of patients with stage IV gastric cancer. J Gastrointest Cancer 2013; 44:199-202.

8. Brar SS, Seevaratnam R, Cardoso R, Yohanathan L, Law C, Helyer L, Coburn NG. Multivisceral resection for gastric cancer with the goal of R0 resection, gastrectomy with MVR can be performed. The benefit of attaining an R0 resection positively influences overall patient survival, even though morbidity and mortality may be higher. Nodal status and the number of organs involved must be taken into account when it comes to patient selection for MVR and an attempt to identify true histological invasion before and during resection should be made.

In locally advanced gastric cancer patients, when a complete resection could be realistically obtained and when lymph node metastatic involvement is not evident, the gastrectomy with MVR should be the therapeutic choice with acceptable postoperative morbidity and mortality rates. Liver, pancreas and the transverse colon resections have proven to be related to increased survival in comparison to MVR with resection of other adjacent structures.

cancer: a systematic review. Gastric Cancer 2012; 15 Suppl $1: \mathrm{S} 100-7$

9. Lim S. Muhs BE, Marcus SG, Newman E, Berman RS, Hiotis SP. Results following resection for stage IV gastric cancer; are better outcomes observedin selected patient subgroups? J Surg Oncol 2007; 95:118-122

10. Kasakura Y, Fujii M, Mochizuki F, Kochi M, Kaiga T. Is there a benefit of pancreaticosplenectomy with gastrectomy for advanced gastric cancer? Am J Surg 2000; 179:237-242.

11. Pacelli F, Cusumano G, Rosa F, et al. Italian Research Group for Gastric Cancer. Multivisceral resection for locally advanced gastric cancer: an Italian multicenter observational study. JAMA Surg 2013; 148:353-360.

12. Li MZ, Deng L, Wang JJ, et al. Surgical outcomes and prognostic factors of T4 gastric cancer patients without distant metastasis. PLoS One 2014; 9:e107061

13. Cuschieri A, Fayers P, Fielding J, et al. Postoperative morbidity and mortality after D1 and D2 resections for gastric cancer: preliminary results of the MRC randomised controlled surgical trial. The Surgical Cooperative Group. Lancet 1996; 347: 995-999.

14. Martin RC 2nd, Jaques DP, Brennan MF, Karpeh M. Extended local resection for advanced gastric cancer: increased survival versus increased morbidity. Ann Surg 2002; 236:159-165. 